\title{
CORRECTION
}

\section{Author Correction: USP29 maintains the stability of cGAS and promotes cellular antiviral responses and autoimmunity}

Qiang Zhang, Zhen Tang, Ran An, Liya Ye and Bo Zhong (iD

Cell Research (2020) 30:821-822; https://doi.org/10.1038/s41422-020-0368-8

Correction to: Cell Research https://doi.org/10.1038/s41422-0200341-6, published online 26 May 2020

We just found several mistakes and typos in the paper published on May 26, 2020. Specifically, the "+" symbols of GFP-USP29 and GFP-USP29(C298A) should be switched in Fig. 6c. The corrected
Fig. 6 is shown here. The "**P<0.001" should be "**P $<0.01$ " in the figure legends of Figs. 2, 3 and 5-7. The word "strained" should be "stained" in the figure legend of Fig. 7. These corrections do not affect the description of the results or the conclusion of this work. We apologize for not detecting them before publication and any inconvenience caused. 
a

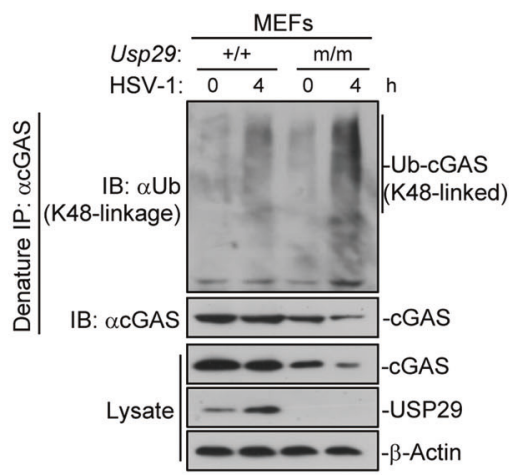

b

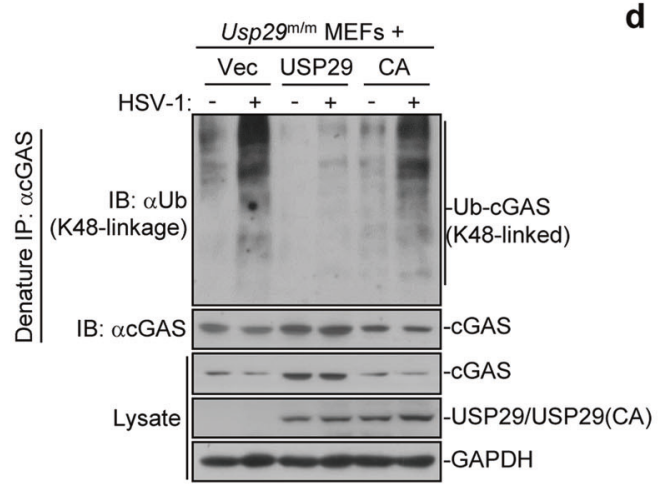

C

d
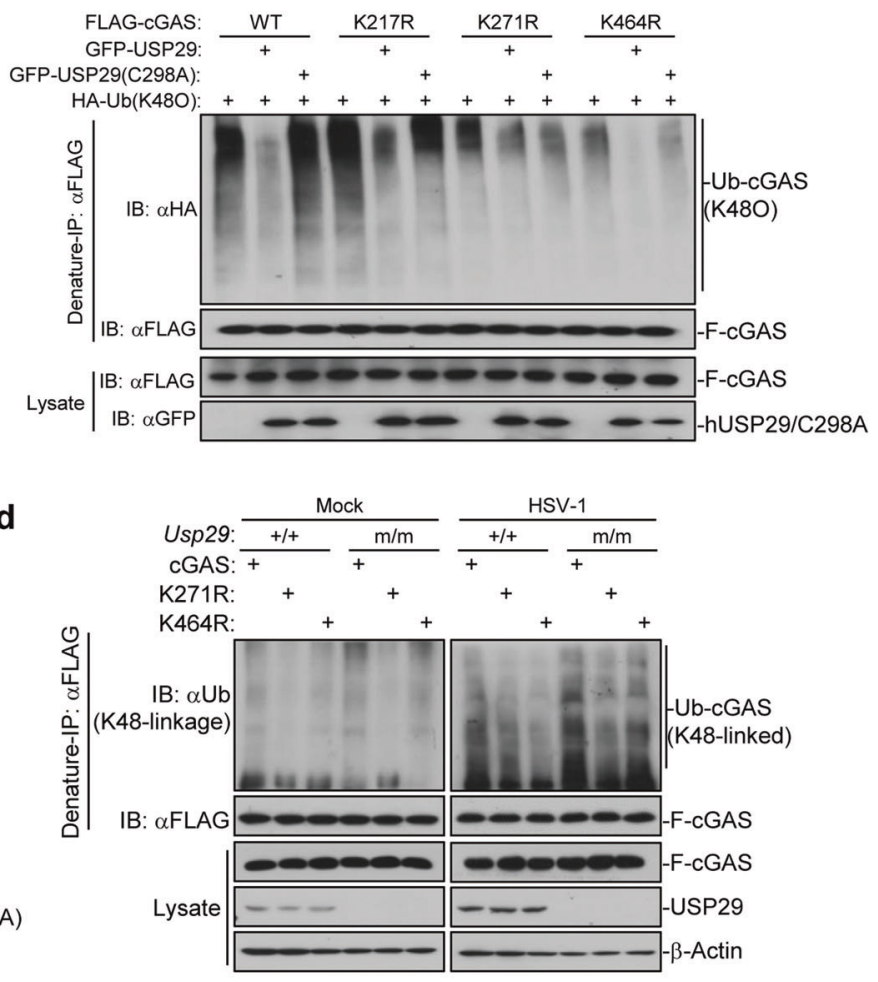

e
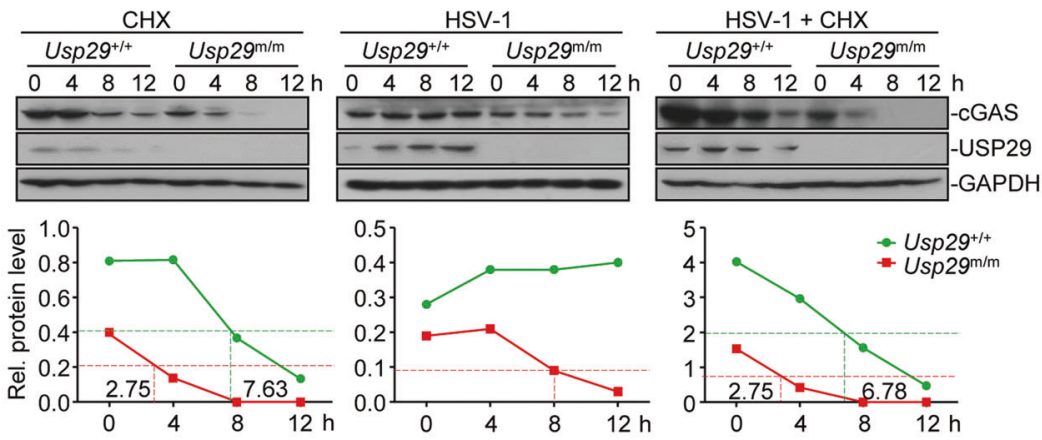

f
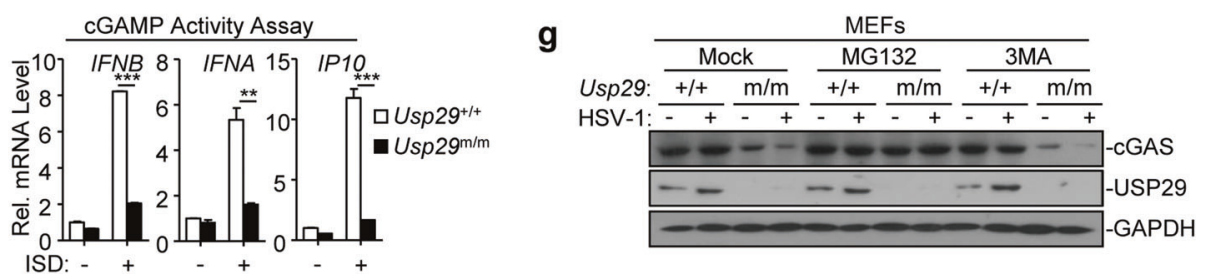

h

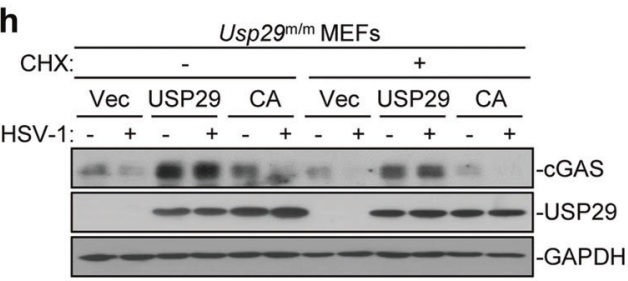

\section{i}

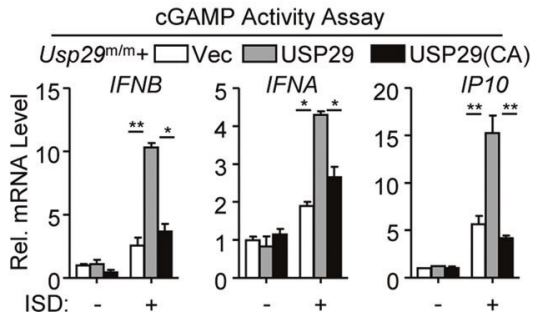

Fig. 6 